\title{
Ethical and Social Challenges in Newborn Screening for Prenatal Alcohol Exposure
}

Can J Neurol Sci. 2014; 41: 115-118

Fetal alcohol spectrum disorders (FASD) are a leading cause of preventable, non-genetic birth defects and intellectual disability in Canada. ${ }^{1}$ Presently, the diagnosis of FASD relies heavily on prior documentation and self-reporting of alcohol use by the biological mother. Measurement of fatty acid ethyl esters (FAEEs) detected in the meconium of a newborn can indicate prenatal alcohol exposure (PAE). The detection of PAE in the newborn serves two important purposes: 1) it provides a quantitative method of identifying children who may be at high risk for developing FASD, and 2) it provides a basis for epidemiological measurements of the prevalence of alcohol exposure during pregnancy. ${ }^{2}$ Ultimately, children who are identified by biomarkers as being exposed to alcohol before birth can be offered early, integrated interventions if symptoms of FASD develop. ${ }^{3}$ Despite the potential health benefits and assurance such tests could provide, they also present significant social and ethical questions for stakeholders (e.g., biological mothers, physicians, foster parents $)^{4}$ as well as important legal aspects. 5 To further examine these questions, we organized an interdisciplinary panel discussion, "Neuroethics and FASD", at the 2012 NeuroDevNet Brain Development Conference in Toronto, Canada. Four Canadian experts spoke at the panel: Dr. James Reynolds, neuroscience researcher, Dr. Gideon Koren, clinical researcher, Anna Zadunayski, LLB, clinical ethicist and lawyer, and Dr. Nina Di Pietro, neuroethicist. Here we report salient discussion points emerging from brief presentations by panelists and subsequent discussion with the audience. We hope that this stimulates further discussion about the social and ethical challenges of biomarker screening for PAE.

\section{Prominent discussion themes}

Five salient topics surfaced: 1) validity of screening methods; 2) disclosure and informed consent; 3 ) populations to screen; 4) best interests, roles and responsibilities of stakeholders; and 5) privacy and the appropriate use of results.

"[Fetal alcohol spectrum disorder] [affects] 1 in 100 babies in this country, it's 4000 new cases of Canadian kids every yearshouldn't we screen everyone to be able to mobilise health, especially in the Canadian context [...]"

Dr. Gideon Koren

\section{Validity of screening methods}

In meconium and maternal hair, FAEEs ${ }^{6}$ measured above a certain threshold have been shown to accurately detect PAE. ${ }^{2}$ However, the actual detection of alcohol exposure is limited by the fact that the fetus does not produce meconium until after the first trimester. Similarly, phosphatidylethanol, another metabolite of ethanol, can be detected quite reliably in the membranes of fetal red blood cells but will only detect alcohol exposure in approximately the last month before birth. Given the asymmetry between true accuracy of alcohol exposure and proper timing of such tests, panellists similarly voiced the need for more research to improve the sensitivity of biomarkers. ${ }^{5}$

"It was in '88 that our laboratory showed first that if mom used cocaine in pregnancy we can find it in the hair of the baby and in the meconium as long term memory of what happened [...] before we get the history."

Dr. Gideon Koren

The dialogue on the methodologies and accuracy of these screens forged the foundation for crucial ethical considerations. Dr. Nina Di Pietro reinforced that, with regards to current biomarker testing, even true positives may not necessarily lead to the development of FASD. Accordingly, she suggested that the implications of false positives have not been fully considered. Dr. Gideon Koren countered these concerns related to false positives by suggesting that these are uncommon, stem from improper application of the test (i.e., time of testing), and that the value of the test resides in the fact that a proportion of those newborns with PAE identified by the test go on to develop FASD. Despite some debate about the predictive potential for current biomarkers, panellists acknowledged the significant value of screening as a way to monitor the development of potential disabilities, to guide the administration of early interventions, and to document the child's prenatal history.

"A biomarker can tell us something about exposure to some compound or chemical substance, in this case, alcohol. But biomarkers can potentially tell us other things, it may be a biomarker of effect, that is some change in a biological system or a behaviour that's a signature of exposure to the chemical compound during development, but probably the most important element of a biomarker would be telling us something about outcomes."

Dr. James Reynolds

\section{Disclosure and informed consent}

Participants brought forth a range of perspectives about the value of an honest and trusting relationship between physicians and patients, which was described as having broad implications from influencing the disclosure of alcohol use by mothers-to-be to altering willingness to consent to screening. For example, an audience member drew attention to the precondition of a safe, supportive environment that is more conducive for women to share their prenatal history. Empathy in the physician-patient setting, suggested Dr. Di Pietro, helps to lessen fear of 
judgement, and even punishment, when seeking prenatal medical care. Early prenatal visits were seen to be important for discussing the issue of drinking during pregnancy and for detecting whether or not a mother-to-be could benefit from intervention. Since many women will not self-report, Dr. Koren noted that it is important to combine self-report with other methods of detection to increase opportunities for intervention which can lead to better outcomes for mother and child.

"[W]ithout exposure history, it's very difficult to make a diagnosis. The question is: do you need permission to do it; do you need mom to say yes to such a test? This is a typical example of maternal fetal conflict, mom may have reasons not to want to do it, such as being embarrassed, guilt feelings, and most importantly, the kid may be taken from her by child protection agencies who may decide that it's not an appropriate environment."

\section{Dr. Gideon Koren}

Consent to screening for PAE is a controversial issue. Should parents need to explicitly express consent for PAE biomarker testing or should screening be carried out based upon implied consent (often the case in other screens for newborns)? Dr. Koren questioned whether or not parental permission should be required for screening, considering that there are long term benefits for the child and that tracking a child's exposure history was deemed vital for making any future diagnosis. Dr. Reynolds alluded to the fact that adopted children with suspected FASD who have no records of prenatal history may specifically benefit from the implementation of a broad (meaning implied) screening program. Anna Zadunayski remarked that women would generally consent to meconium screening for PAE, although they were uncertain if it was going to make a difference for their child, how the information would be used, and who would have access to it. Under the implied consent model, parents have to opt out of the screening but Dr. Di Pietro indicated that they are often unaware of the tests that are being administered, and the fact that they can opt out at all. Open and early discussions between physicians and patients about FASD, the screening procedure, possible outcomes and actions can foster a mutually honest relationship in which physicians understand what shapes patients' preferences, and where patients are well informed of the choices and outcomes of screening.

"[S]tudies interviewing families about their knowledge of what kind of screening is going on [...] have shown that basically parent [s] quite often aren't even aware of what kind of screening is being done on their newborn baby, and many of them are not aware that they can even opt out of this kind of screening, so if we were to include screening for prenatal alcohol exposure I would argue that given the sensitive nature of the information involved and the impact [...] on the families and the potential for stigma, that this kind of screening really shouldn't follow that kind of implied consent model. That instead we should offer the parents a chance to actually opt in with informed consent, sit down with them, tell them about what kind of screening is going to be done and what are the potential implications to them, and have them opt into that rather than simply opt out." (Quote slightly edited for clarity)

\section{Populations to screen}

Discussions around different target populations for screening programs incited deliberation about the benefits and drawbacks of targeted and universal screening. According to Dr. Di Pietro, it is presently ethically unfeasible to provide targeted screening in hospitals, for fear of inducing stigma and stereotyping of groups determined to be "at risk". Moreover, how would characteristics of an "at risk" population be determined? In contrast, these tests in a universal fashion will capture purportedly "at risk" groups and although it may increase cost of the screening programs, it could reduce outward discrimination towards these groups. It is important to note that Dr. Koren's work has found that screening is cost effective for communities with higher prevalence of FASD, when considering the long term benefits and lifetime savings. ${ }^{7}$

\section{Best interests, roles and responsibilities of stakeholders}

It was clear that a constructive conversation about screening needed to keep in mind the players involved: the individuals receiving the test (both mother and child), the medical practitioners, as well as communities and governments. However, the interests of these parties may conflict. These facts significantly challenge the roles and responsibilities of stakeholders. For example, the "maternal-fetal conflict" is illustrated in the example where a woman may be loath to undergo alcohol screening out of embarrassment or guilt, particularly if there is a chance that her child could be taken from her if results are positive. Punitive policies in screening are not in the best interests of either the mother or infant and they do not necessarily prevent women from continuing to abuse alcohol or drugs. Anna Zadunayski suggested that an ethical argument in favour of screening would be that it acts in the best interests of the child who has potentially been exposed in order for the results to be properly documented and used to assist physicians in providing appropriate care throughout development. Clearly, different groups are intertwined in a network of obligations to themselves and to others; a relevant aim is to elucidate harms and minimize them through broader dialogue on screening.

"[I]t's time to have a national conversation around what policy guidelines should be in place, so that we can use the screening to its absolute benefit [so] that we can help children, but that we're not alienating another population from the healthcare system at the same time."

Anna Zadunayski, LLB

\section{Privacy and the appropriate use of results}

The health benefits of screening compete with social and legal implications, including privacy, intended use, and clinical practice guidelines. Thus, not only should medical and governmental stakeholders be consulted, but so should legal professionals, chiefly because the scientific means and knowledge to carry out tests exist, in the absence of relevant laws and policies. Anna Zadunayski reported that results of meconium testing have been used in divorce and custody cases and in criminal law (e.g., admitted evidence to argue against the mother's integrity). These examples indicate the need to (reeducate) legal stakeholders, and to advocate for potential legal 
Table 1: Examples of the questions raised by the panellists and audience members with regards to ethical and social issues in newborn screening for biomarkers of PAE

What kinds of healthcare resources/intervention programs currently exist for FASD, and how effective are they?

What are the challenges of clinical follow-up for different Canadian populations (e.g., rural or urban) and their impact on the trade-off between risks and benefits?

Should parental consent be required for screens? Or should meconium screening be included with other newborn screens such as those for phenylketonuria (PKU), such that implied consent takes the place of informed consent?

Should screening be available to everyone? If not, what clinical and ethical criteria should be used to determine which mothers should be screened?

Are the ethical challenges of consent and privacy currently outweighed by the potential clinical value of biomarkers of prenatal alcohol exposure in Canadian children?

How will Canadian courts interpret findings of prenatal alcohol exposure? What guidelines will be put in place to ensure that misinterpretations are minimized?

What, if any, information should be disclosed to third parties such as social services? How can non-punitive policies be implemented?

How can we start a national conversation on the use of biomarkers of prenatal alcohol exposure?

clarification on the interpretation of testing results, especially when the application of the screen is time-sensitive to ensure accuracy. As a result of the absence of specific privacy protections, healthcare systems may alienate women (especially those who are more at risk for substance abuse) and dissuade them from giving birth in medical settings with medical professionals.

"If a positive screening result is obtained, is it in the best interest to keep that information in the medical arena to provide services to the families, or should we or shouldn't we report this data to perhaps child welfare agencies, I think that's another major issue up for debate."

Dr. Nina Di Pietro

\section{Questions and Recommendations}

The emergence of new technology inevitably produces concerns about its intended use and the existence of appropriate regulations. An interdisciplinary group of panellists in research, clinical, ethical and legal fields brought these underdeveloped social, legal and ethical issues to the surface (Table 1) and provided some critical recommendations (Table 2) about screening for PAE. The intense discussion we witnessed among our panel and the public suggests that further reflection and research is required on the topic of development and implementation of biomarkers in PAE.

Table 2: Proposals made by the panellists and audience members with regards to ethical and social issues in newborn screening for biomarkers of PAE

Encourage positive and safe environments for women to honestly discuss alcohol use, prenatal history and FASD prevention with their physicians

Respect confidentiality, and implement non-punitive policies for screening

Acknowledge the usefulness of results of screening as indicators of prevalence, and inform the government where resources should be allocated, and how informed people are about health behaviours during pregnancy

Support a fully informed consent process for PAE screening 


\section{ACKNOWLEDGMENTS}

Support from this work comes from NeuroDevNet (Racine), and the Canadian Institutes of Health Research; New Investigator Award (Racine). The authors thank the members of the Neuroethics Research Unit for feedback on a previous version of this manuscript, to Bethany Becker for help with the audio recording, and to NeuroDevNet for general support.

Allison Yan, Emily Bell, Eric Racine Institut de recherches cliniques de Montréal (IRCM) Montreal, Quebec, Canada

\section{REFERENCES}

1. Public Health Agency of Canada. What is fetal alcohol spectrum disorder (FASD)? Public Health Agency of Canada, Ottawa, ON, 2007, Available at: http://www.phac-aspc.gc.ca/hp-ps/dcadea/prog-ini/fasd-etcaf/publications/pdf/factsheet1-fasd-etcafeng.pdf, accessed August 6, 2013.

2. Joya X, Friguls B, Ortigosa S, et al. Determination of maternal-fetal biomarkers of prenatal exposure to ethanol: a review. J Pharm Biomed Anal. 2012;69:209-22.

3. Canadian Association of Paediatric Health Centres. National screening tool kit for children and youth identified and potentially affected by FASD, Canadian Association of Paediatric Health Centres, Ottawa, ON, 2012, Available at http://ken.caphc.org/xwiki/bin/download/FASDScreeningToolki t/National+Screening+Tool+Kit+for+Children+and+Youth+Ide ntified+and+Potentially+Affected+by+FASD/FASDToolKitBoundEN.pdf, accessed August 6, 2013.

4. Zizzo N, Di Pietro N, Green C, Reynolds J, Bell E, Racine E. Review and reflections on ethics in screening for biomarkers of prenatal alcohol exposure. Alcohol Clin Exp Res. 2013 Sep;37 (9):1451-5.

5. Dickens BM. Legal and ethical considerations in meconium testing for fetal exposure to alcohol. J Popul Ther Clin Pharmacol. 2011; 18(3):e471-4.

6. Koren G, Hutson J, Gareri J. Novel methods for the detection of drug and alcohol exposure during pregnancy: implications for maternal and child health. Clin Pharmacol Ther. 2008;83(4): 631-4.

7. Zelner I, Koren G. Universal or targeted screening for fetal alcohol exposure: a cost-effectiveness analysis. Ther Drug Monit. 2009; 31(2):170-2. 